\title{
Design of an Augmented Reality Magnification Aid for Low Vision Users
}

\author{
Lee Stearns \\ University of Maryland \\ College Park, MD, USA \\ 1stearns@umd.edu
}

\author{
Leah Findlater \\ University of Washington \\ Seattle, WA, USA \\ leahkf@uw.edu
}

\author{
Jon E. Froehlich \\ University of Washington \\ Seattle, WA, USA \\ jonf@cs.washington.edu
}

\begin{abstract}
Augmented reality (AR) systems that enhance visual capabilities could make text and other fine details more accessible for low vision users, improving independence and quality of life. Prior work has begun to investigate the potential of assistive AR, but recent advancements enable new AR visualizations and interactions not yet explored in the context of assistive technology. In this paper, we follow an iterative design process with feedback and suggestions from seven visually impaired participants, designing and testing AR magnification ideas using the Microsoft HoloLens. Participants identified several advantages to the concept of head-worn magnification (e.g., portability, privacy, ready availability), and to our AR designs in particular (e.g., a more natural reading experience and the ability to multitask). We discuss the strengths and weaknesses of this AR magnification approach and summarize lessons learned throughout the process.
\end{abstract}

\section{Author Keywords}

Low vision; visual impairment; assistive technology; wearables; augmented reality; magnification.

\section{ACM Classification Keywords}

- Human-centered computing $\rightarrow$ Accessibility technologies

- Computing methodologies $\rightarrow$ Mixed / augmented reality

\section{INTRODUCTION}

Recent advancements in augmented reality (AR) have the potential to increase the quality of life for people with visual impairments (VI). For low vision users, headmounted displays (HMDs) that enhance a user's existing visual capabilities are particularly promising. For example, ForeSee [25] used an Oculus Rift VR headset with an attached camera to magnify and enhance text content, and other researchers have used Google Glass to enhance edges within the wearer's field of view [9] or display magnified

Permission to make digital or hard copies of all or part of this work for personal or classroom use is granted without fee provided that copies are not made or distributed for profit or commercial advantage and that copies bear this notice and the full citation on the first page. Copyrights for components of this work owned by others than the author(s) must be honored. Abstracting with credit is permitted. To copy otherwise, or republish, to post on servers or to redistribute to lists, requires prior specific permission and/or a fee. Request permissions from Permissions@acm.org.

ASSETS '18, October 22-24, 2018, Galway, Ireland

(C) 2018 Copyright is held by the owner/author(s). Publication rights licensed to ACM.

ACM ISBN 978-1-4503-5650-3/18/10 \$15.00

https://doi.org/10.1145/3234695.3236361

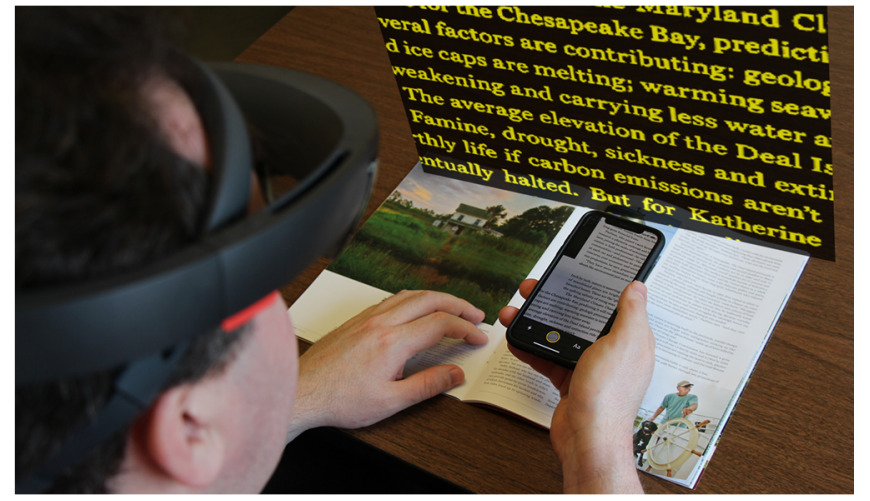

Figure 1. Prototype AR Magnification system using a transparent HMD (the Microsoft HoloLens) and a handheld smartphone (iPhone $X$ ) as a camera and input device.

content from a smartphone screen [16]. Several commercial HMDs (e.g., eSight [27], NuEyes [28], IrisVision [29]) display magnified video captured from a head-mounted camera, and provide image enhancement features such as contrast adjustment. A recent study investigating the use of one of these systems (eSight) was generally positive, showing the impact HMDs can make in users' lives [26].

While these systems have begun to explore how HMDs and wearable cameras can be used to augment visual perception, they are limited to enhancing and/or magnifying the $2 \mathrm{D}$ image from a video camera. In contrast, the classical definition of $A R$ integrates $3 \mathrm{D}$ virtual objects into the $3 \mathrm{D}$ physical environment [1], which would allow for new visual enhancement possibilities that are better integrated with the user's real-world tasks. For example, a magnified view of an object can be rendered directly on top of the real object, fixed to a desk near the user's primary work focus, or "projected" on a nearby wall. Off-the-shelf technologies such as the Microsoft HoloLens [30], an optical see-through display, are beginning to have the capability to support these types of 3D AR designs.

To investigate the design possibilities for AR magnification tools enabled by registering virtual content in real 3D space, we conducted a series of design sessions with seven lowvision participants. We developed initial prototype designs on a Microsoft HoloLens, which we presented to participants to solicit feedback and open-ended ideas about future wearable magnification aids. Our designs explored several different virtual display options (e.g., affixed to real objects $v s$. moving with pointing finger), image acquisition 
methods (head-mounted, finger-mounted, or smartphone), and interaction techniques (e.g., Figure 1). The designs were updated between sessions based on participant feedback as well as our own observations.

Overall, participants liked the concept of a wearable AR magnification aid, especially the natural reading experience and ability to multitask that the projected 3D renderings enabled. At the same time, our system presented some difficulties compared to participants' existing magnification aids. We discuss these issues along with potential solutions and design implications.

Our contributions include: (i) an exploration of the design space for augmented reality magnification; (ii) proof-ofconcept implementations evaluated and refined through iterative design with low vision users; and (iii) common themes and recommendations that should inform the design of future $\mathrm{AR}$ vision enhancement aids for low vision users.

\section{RELATED WORK}

Our research is informed by prior work on magnifiers, AR systems, and wearables designed for low vision users.

\section{Low Vision Magnification Aids}

Common magnification aids for low vision users include low-tech optical magnifiers, desktop video systems (e.g., $[31,32]$ ), handheld devices (e.g., [33,34]), and more recently mobile applications that use a smartphone's camera and display (e.g., [35]). Both Virgili et al. [23] and the American Foundation for the Blind [2,3] provide a comprehensive overview of these options.

Desktop video magnifiers, also known as closed-circuit television (CCTV) systems, project images from a stationary or handheld camera onto a large television or computer monitor. Some examples are the ClearView $+H D$ [31] and the DaVinci HD [32]. These systems benefit from a large screen and ready customization, allowing users to adjust the positioning, magnification level, and other features for a comfortable reading experience. However, they are not portable and the magnified display is separated from the actual content, which could cause difficulty when reading longer passages or searching for a specific location at higher magnification levels. To address this latter issue, a conventional optical magnifier or other digital magnification aid is sometimes used to locate a target before reading it with the CCTV [4].

Handheld video magnifiers are a portable alternative that can be used in a greater variety of situations (e.g., to identify items on a shelf). For example, Ruby [33] and Compact $+H D$ [34] both function as a magnifying glass with image enhancement capabilities. Dedicated hardware can be optimized for the needs of low vision users (e.g., large, high-contrast components and controls) and physical buttons allow for adjustment of common settings (e.g., brightness and contrast). However, most of these systems are designed for a single purpose, so users may need to carry multiple devices to identify both near and distant text or other objects, in addition to other general devices such as smartphones. Also, despite theoretically being portable, many of these devices are still bulky, necessarily occlude the physical object being magnified, and may need to be held in such a way that viewing the magnified image cannot be done discreetly.

Smartphones also increasingly support magnification for low vision users. Apple and Android phones now include a built-in magnifier $[35,36]$ and numerous free or low-cost third-party apps are available in the Apple or Google stores. Unlike dedicated handheld magnifiers, these smartphonebased solutions are cheaper, do not require carrying an additional device, and are likely to draw less attention to the user. However, they present other similar disadvantages to dedicated handheld magnifiers.

Our research builds on these designs, using augmented reality to provide a portable and perceptually large virtual display with common image enhancements (e.g., brightness, contrast, color alteration) that can be used to identify text and other fine details, both near and far.

\section{HMDs to Enhance Vision Capabilities}

Head-worn vision enhancement systems for low vision users were first proposed in the 1990s [7,12]. Compared to other types of low vision aids, HMD-based solutions offer potential advantages of portability, ready availability, and privacy while displaying enhanced information within the wearer's field of view. A recent study by Zolyomi et al. [26] showed that one such device (eSight [27]) improved access to information and social engagement but also had negative social impacts [26]. Another study by Profita et al. [15] investigated the social acceptability of HMDs, showing greater acceptance if the device is being used for an assistive purpose as opposed for a general mobile computing task.

Most HMD-based systems for low vision users project magnified and/or enhanced 2D video captured from a wearable camera onto screens mounted in front of the user's eyes [12,25,27-29]. Some recent examples have used consumer VR hardware: ForeSee [25] uses an Oculus Rift headset and IrisVision [29] uses a head-mounted smartphone (Samsung GearVR). Optical see-through displays have also been employed for vision enhancement $[9,16]$, where virtual information is overlaid on a transparent display, thus augmenting rather than replacing the user's vision. For example, Google Glass has been used to display a magnified view of a smartphone screen [16] and to overlay enhanced edges onto the wearer's view of the real world [9]; however, Glass itself is a low-resolution display $(640 \times 360)$ and not designed as an AR device (e.g., the display is positioned in the user's visual periphery).

None of the above solutions have overlaid virtual content in 3D space - our focus. However, Zhao et al. [24] conducted an accessibility evaluation of the Epson Moverio BT-200 smart glasses with participants with low vision. They 
concluded that while the semi-transparency of optical seethrough displays did reduce contrast and make it somewhat harder for low vision users to read text or identify shapes, participants were able to successfully use the device and were positive about the experience, confirming that devices like the HoloLens are a useful prototyping platform for our investigation. In a recent ASSETS poster [20], we presented three early designs exploring how the HoloLens might support low vision users by enhancing an image captured by a finger-mounted camera: showing a magnified view of the camera image at a fixed position in the wearer's view, affixing the image to a 3D surface, and having the image track the user's finger. While illustrating some possibilities for true AR vision enhancement, that work was preliminary did not include any evaluation or feedback from low vision users. Our current research builds on these early explorations, exploring the capabilities of the HoloLens as an AR magnification aid and evaluating several designs with low vision users.

\section{Other Assistive Wearables for Low Vision Users}

Our work also builds on other research on assistive wearables for blind and low vision users. The majority of these tools are designed for users with a wider range of visual abilities, including no functional vision, and so do not include a display. Information is primarily conveyed using text-to-speech and other audio cues.

OrCam [14] is a commercial head-worn camera system (with no visual display) that users control with speech and gestures. The system can identify and read printed text using text-to-speech and can identify pre-trained products and faces. Other systems use cameras worn on the head [11] or wrist [19] to identify faces, and an early version of Microsoft's recent Seeing AI smartphone app [37] used a pair of smart-glasses to identify people, describe facial attributes, and read text, and was controlled by touch gestures on the side of the glasses [38]. Finger-mounted cameras have also been used to support visually impaired users in a variety of tasks, including reading printed text via text-to-speech output $[18,21]$, controlling mobile devices [22], and identifying currency or other objects [13].

While our work differs from these systems in application and in some cases target user group, we also explore tradeoffs between wearable/portable camera locations, including head-worn, finger-worn, and handheld.

\section{A DESIGN SPACE FOR MAGNIFICATION AIDS}

To inform the design of an AR magnification aid for low vision users, we first outline our goals and important design dimensions for mobile and/or wearable magnification aids.

\section{Design Goals}

Informed by prior work, existing commercial systems, and our own experience working with initial AR prototypes, we formulated the following design goals for our study:
- Augment rather than replace. Whenever possible, avoid interfering with the user's existing vision capabilities. Provide enhanced content alongside the real world and provide easy controls to hide or reposition the digital information as needed.

- Leverage augmented reality. Go beyond the static 2D displays provided by existing systems and explore applications for persistent digital content overlaid in 3D onto the physical world.

- Prioritize customization and flexibility. To support a wide range of vision levels and different situations, the ability to customize how the enhanced content functions and appears is crucial [10].

\section{Design Dimensions}

To achieve these goals, we considered several design dimensions in addition to virtual display position, our primary dimension of interest:

- Virtual display position. The ability to anchor virtual content to a physical location in 3D space enables several possible virtual display designs. Specifically, we explore four positions. The first, simplest position is a fixed heads-up display that moves with the user's head to always stay within their field of vision. The second position is a stationary display attached to a location in the physical world, which maintains its position as the user moves. The third option is a dynamic display that acts as a magnifying glass and follows the user's hand or other moveable object (e.g., a ring or smartphone). Finally, the fourth position projects an image directly onto the physical object that is being enhanced (e.g., a magnified view shown atop a document).

- Content capture. To capture video for processing and display, possible camera locations include head-worn (e.g., [14,25,27-29]), hand-held (e.g., [33,34,37]), and finger or wrist-worn (e.g., ring or smartwatch; [18-20]). We explore a few of these options and discuss the advantages and disadvantages of each.

- Image enhancements. To support a range of vision levels, important enhancements include magnification, changes to brightness and contrast, binary thresholding, and color alterations (e.g., as described in [25]). Although not the focus of our study, optical character recognition could also be useful, either to read text aloud or to visually enhance detected text by increasing the resolution or replacing fonts.

- Physical HMD. Several display types have been explored previously, as discussed in the Related Work section. However, an optical see-through display and 3D sensing capabilities are needed to achieve our design goals, making the Microsoft HoloLens the obvious choice at the time this research was conducted. The HoloLens allowed us to rapidly prototype and iterate on AR designs; however, we fully expect that future HMDs for AR will be more streamlined, lightweight, and portable (e.g., integrated into traditional glasses). 
- User input. To support our goal of customizability and flexibility, the AR system needs to provide intuitive and easy-to-use interactive controls. A few options include physical controls on the device or a separate remote (e.g., eSight [27], Glass [5]), gaze tracking, midair gestures, and voice commands (e.g., OrCam [14], HoloLens [30]), or 3D tracking of a physical object (e.g., Oculus Rift controller [39]). We explore a few of these options to see how well they work for low vision users and in different situations.

\section{ITERATIVE DESIGN OF A PROTOTYPE SYSTEM}

To explore these design dimensions and evaluate which designs and features would work best for low vision users, we conducted a series of iterative design sessions. These sessions were structured to elicit general feedback and open-ended design ideas from participants, drawing on elements of cooperative [6] and participatory [17] design methodologies. Based on ideas from existing magnification aids, knowledge of available hardware capabilities, and our own experience working with low vision users, we developed an initial prototype system that implemented several user interface designs. We then asked participants to use the system and provide feedback, refining our design over nine design sessions with seven unique participants (two participants returned for a second session). While we modified the system between sessions to fix issues and make minor improvements, for ease of presentation, we group our prototypes into three basic designs based on the broad design elements, components, and user interactions.

\section{Initial Investigation: HoloLens Only}

Our initial design used only the HoloLens headset. As mentioned earlier, the HoloLens includes an optical seethrough display on which translucent virtual objects ("holograms") can be overlaid in real 3D space. The estimated field of view is $30^{\circ} \times 17.5^{\circ}$ with 2500 light points per radian. Microsoft's APIs include motion tracking and 3D scene analysis functions that allow developers to anchor digital content to a physical location in the world so that it will remain stationary as the user moves. The APIs also support input using midair gestures, the direction the user's head is pointing, and voice.

This initial prototype used the HoloLens's built-in camera to capture images of what the user looked at and provided two modes for displaying an enhanced version of those images: fixed 2D and fixed 3D. While we describe these display modes in more detail in the next section, the fixed 2D display moved with the user's head to always remain within view while the fixed 3D mode was anchored to a surface in the physical world. Users could toggle between modes using voice commands and two image enhancement options were provided: binary thresholding (black text on a white background) and color inversion.

While this initial design was functional, internal testing revealed that the HoloLens' built-in camera resolution was simply too low to be useful when magnified. Additionally,

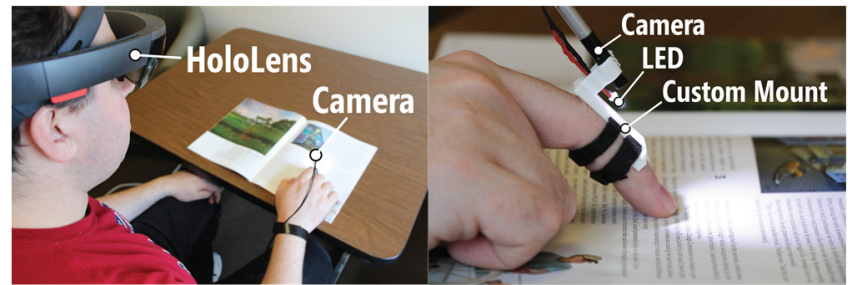

Figure 2. First prototype system design: (a) full system with the HoloLens, (b) close-up of the finger-worn camera

requiring users to turn their head to look at desired content for magnification was uncomfortable, and the voice commands were cumbersome and imprecise. We used these observations to develop the next iteration of our prototype, which was the first to be tested with low vision participants.

\section{Prototype 1: HoloLens and Finger-Worn Camera}

To address the issues observed in our initial investigations and to expand on our design, we added an external camera, implemented two additional display modes and more customization options, and replaced the voice commands with a virtual menu controlled using midair gestures. We then conducted design sessions with three participants, making minor changes to the system between sessions based on feedback (e.g., modifying the perceptual distance at which the AR displays were drawn, simplifying and polishing user input).

\section{Implementation Details}

Below we describe the prototype's components and physical design, the four display modes, and the user interactions.

Hardware and Physical Design. This prototype used an external camera ${ }^{1}$ mounted on the user' finger using a custom 3D-printed ring with Velcro straps and an LED to provide consistent lighting (Figure 2). The camera provided a close-up view of the target content and allowed the user to read without needing to frame the text within the head-worn camera's field of view. As discussed earlier, similar wearable cameras have been used for other assistive devices $[13,18,21,22]$, albeit primarily for people with more severe visual impairments. We used a laptop computer to capture and process images from the camera, which we streamed wirelessly in real-time to the HoloLens for display.

Virtual Display. To elicit feedback on a range of AR display designs, we implemented four display options for the enhanced view from the camera, including the two explored in the initial prototype (Figure 3 and video figure):

- Fixed 2D: This design displayed the image at a fixed location relative to the user's head, which meant that the display was within the user's view at all times. This design is similar to past work using HMDs for visual enhancement (e.g., $[9,25,27])$, and it is possible to implement on more basic HMDs such as Google Glass.

\footnotetext{
${ }^{1}$ Awaiba NanEye GS Idule Module, $640 \times 640 \mathrm{px}, 30^{\circ} \mathrm{FoV}$
} 


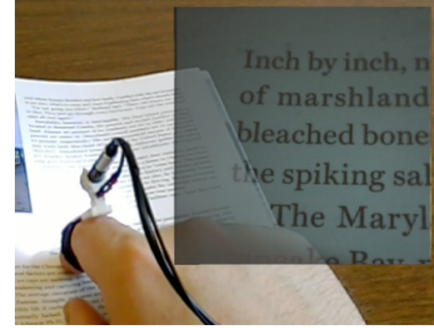

(a) Fixed 2D

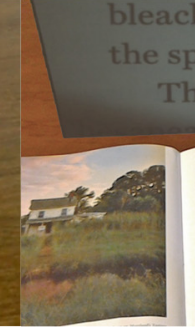

(b) Fixed 3D (Vertical)

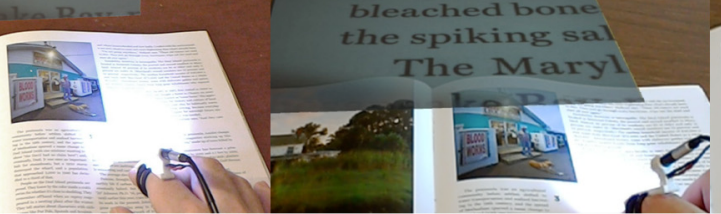

(c) Fixed 3D (Horizontal)

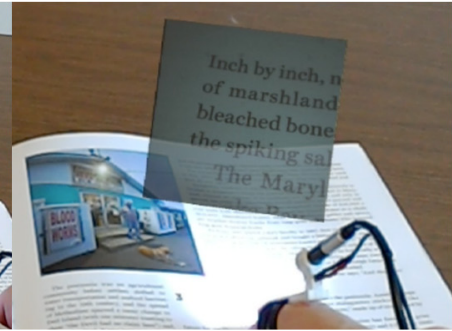

(d) Finger Tracked

Figure 3. Prototype 1 provided four virtual display modes, which could be customized (position, size, zoom) using midair gestures. See the accompanying video figure for a demonstration.

- Fixed 3D Vertical: This design allowed the user to place the enhanced view from the video camera at a fixed position in the physical world. The display was oriented vertically, allowing for placement on a vertical surface like a wall. It remained at the fixed location as the user turned their head or moved around.

- Fixed 3D Horizontal: This design was similar to Fixed 3D Vertical but the display was oriented horizontally so it could be placed on a tabletop or other flat surface.

- Finger Tracked: In this design, the display was oriented vertically above the user's finger and moved along with the user's hand like a magnifying glass. Because the HoloLens APIs could not detect the user's hand when touching a page, we used a simple image processing technique to detect the bright LED from the finger-mounted camera and position the display near it.

As with the previous prototype, the system provided image binarization and color inversion features, which participants could use as desired. The brightness of the LED and the HoloLens display could also be adjusted.

User Interactions. Because voice commands proved too limiting for the variety of customization options we wanted to support, we used the gesture recognition capabilities provided by the HoloLens to allow users to adjust the display's position, size, and zoom level. Users opened the virtual menu using the default HoloLens "air tap" gesture, and selected from three options (position, size, or zoom level) by turning their head to position a cursor atop the desired item and then performing another air tap gesture. To move or adjust the display, users performed a "manipulation" gesture, first lowering their index finger, moving their hand in any direction, then raising their finger again once satisfied.

The first two VI participants found these interactions to be difficult, so we reduced the number of menu items (leaving only position and size) and added remote controls to allow us adjust settings as directed by the participant if needed.

\section{Design Sessions}

Three participants used our first prototype, comparing the display modes and discussing the overall idea of augmented reality vision enhancement.

Participants. We recruited three participants who used a CCTV or other magnification aid in their daily lives (two male, one female, age range 28-54). The cause and severity of participants' visual impairments varied (Table 1).

Methods. After an open-ended interview to collect demographic information and participants' experience with magnification aids, we introduced our system and demonstrated its features. Participants then used each of the four display modes in a partially counterbalanced order (using 3 out of 4 orders from a balanced Latin square) to read text on a variety of objects, including simple printed documents as well as mail, a pill bottle, and a box of cereal. After each mode, participants provided feedback on their likes and dislikes for that particular mode, as well as thoughts about the customization options. At the end of the study, we asked about experiences using the system and which display modes were most and least preferred, discussed the overall design of the system, and elicited

\begin{tabular}{cccccccc} 
ID & S1 & S2 & Age Gender & Diagnosis & $\begin{array}{c}\text { Visual Acuity } \\
\text { (self-reported) }\end{array}$ & Visual Field \\
\hline P1 & $\checkmark$ & $\checkmark$ & 28 & M & LHON & 20/400 or 20/450 & Limited central vision \\
\hline P2 & $\checkmark$ & & 46 & F & Retinitis pigmentatosa & Low vision (acuity unknown) & Limited \\
\hline P3 & $\checkmark$ & $\checkmark$ & 54 & M & Optic atrophy (meningitis) & 20/200 & Full \\
\hline P4 & & $\checkmark$ & 29 & F & Tumor & Low vision (acuity unknown) & None in left, tunnel vision in right \\
\hline P5 & $\checkmark$ & 58 & M & Cone-rod dystrophy & Light and shapes (acuity unknown) & Limited central vision \\
\hline P6 & $\checkmark$ & 33 & F & Oculocutaneous albinism & 20/400 in good lighting & Full \\
\hline P7 & $\checkmark$ & 68 & F & High myopia & 20/400, better in ideal conditions & Full, but better peripheral vision \\
\hline
\end{tabular}

Table 1. Demographic information for the participants across all design session. Columns "S1" and "S2" indicate participation in design sessions with prototype 1 and prototype 2 , respectively. 
suggestions for improvements and new features. Each session lasted approximately two hours, and participants were compensated $\$ 60$ for time and travel costs.

Overall Response and Display Modes. The participants each used the system to read the provided materials, with varying levels of success. P1 and P3 reacted positively to the concept of a wearable AR magnifier. P1 commented:

"If there was something I could just wear and then be able to see something better, point the camera at it and then have it right there in front of my eyeball then I would use that all the time... You could certainly do many things that you're not able to do by yourself at this point."

Both P1 and P3 observed advantages to the 3D design elements incorporated into three of the display modes. They considered the two fixed 3D display modes to be more like the reading experience with a CCTV or handheld magnifier than the other two designs, while the dynamic finger tracking design could potentially help to quickly locate a particular location in a document.

Overall, P1 preferred the two fixed 3D designs (vertical or flat) because they were steadiest and easiest to read, while P3 preferred the fixed 2D design because it was always visible and required the least concentration to use. In contrast to the other two participants, $\mathrm{P} 2$ found the reading process too difficult and did not see advantages to the AR magnification approach, stating that she would prefer to use audio output from a screenreader for most reading tasks.

All three participants disliked the dynamic finger tracking display, primarily due to technical issues with our implementation. This design required participants to turn their head to look directly at their finger, which had two problems: first it was uncomfortable and required additional concentration to keep their finger always within the HoloLens camera's field of view, which interfered with the reading experience. Second, the bright LED reduced the contrast of the virtual display and made it more difficult to read the enhanced text. Interestingly, P1 also found the Fixed 2D display to be difficult to use because its perceptual distance was fixed too far away-we made this setting adjustable for future participants.

Finger-worn Camera. Perceptions of the finger-worn camera were also mixed. The camera was wearable, which allowed for hands-free use, and separation from the display provided flexibility to allow participants to find a comfortable reading position. However, participants disliked the need to move their finger to read (P2) or found it difficult to move from one line to the next for longer passages (P3). The biggest limitation was the small field of view due to the camera's proximity to the page - each image contained only 3-4 lines of text and a few words across. This problem was compounded by the limited field of view of the HoloLens, which when magnified to a readable size sometimes meant that participants could only fit a word or two on the display at a time. All three participants mentioned that their existing magnification aids had a similar problem, but also stated that our system was worse in its current implementation.

The HoloLens Device. Participants' other feedback about the prototype system primarily centered around limitations of our chosen hardware, especially the physical size, weight, and display contrast. Contrast was a source of difficulty for all three participants, to varying degrees. Images displayed on the HoloLens screen are translucent, which meant that text could be difficult to recognize depending on the background imagery. This issue was addressed somewhat by lowering the room lighting or moving the display so that it was positioned over a flat, high contrast surface (e.g., a white wall or black screen). As mentioned above, the bright LED interfered with reading, so the participants mostly positioned the virtual displays so that they were not looking directly toward it. Even with these measures, P2 was unable to successfully use the system to read because of how the HoloLens display functioned, only able to make out a few scattered words and letters. This finding fits with previous mixed results using optical see-through displays for low vision users [24], and suggests that the HoloLens may work better for some types of visual impairment than others.

User Input. While P1 and P3 were able to use the midair "air tap" gestures to adjust the display, all three participants found the gestures to be cumbersome and difficult to use. We frequently needed to provide assistance when changing settings. Because of these difficulties, participants may not have fully customized the system to meet their specific needs. Additionally, the combination of the slow input gestures and the camera's physical positioning meant that participants could not quickly adjust the magnification level to help with locating the start of a new line or another desired location in a document.

Technology Comparisons. When asked to compare the device with their existing magnification aids, all participants stated that the current version was less convenient, primarily due to limitations with the physical hardware. However, if those issues could be solved, P1 stated:

"In comparison to the portable CCTV I have or the full
size one, this would be something you could wear and
take with you... If you just have a pair of glasses that
could essentially do the same thing [as a phone] then I
would probably use that even more than my phone."

Summary. Two participants reacted positively to the idea of AR magnification and observed potential advantages to our 3D display modes. Hardware and user interface issuesespecially the field of view, image contrast, and midair gestures - limited the usability of our prototype, with one participant unable to use the system to read at all. Despite these issues, the overall concept showed promise. 


\section{Prototype 2: HoloLens and Smartphone}

To address these Session 1 study findings, we redesigned several aspects of our system (detailed below). We then recruited six participants for further design sessions using the updated prototype.

\section{Implementation Details}

Below we describe changes to the prototype's components and physical design, display modes, and user interactions. Figure 4 shows the updated prototype in action.

Hardware and Physical Design. Because the finger-worn camera had a narrow field of view and required manual focus, we decided to instead experiment with a handheld smartphone camera (an iPhone X). The smartphone is heavier than the finger-worn camera and does not allow for hands-free usage - a feature of the previous design that participants found appealing - but the change provided a higher-quality camera with built-in processing and wireless communication capabilities. In particular, the camera's high resolution and autofocus allowed users to easily control the amount of text captured by moving the phone toward or away from the page. Users could also adjust the brightness of the phone's camera flash LED to help with contrast. We imagine that a future wearable device (e.g., ring or smartwatch) could incorporate these features as well, if they proved useful for applications like this one.

The use of a smartphone also enabled several new user interactions to control the display settings using the touchscreen and motion sensors, which we discuss below. The phone connected wirelessly with the HoloLens to stream video, touchscreen gestures, and 3D motion data.

Virtual Display. Our updated prototype provided three display modes (Figure 5; video figure), which were refined versions of the four tested previously. We differentiated the modes by the object to which the display was attached:

- Attached to Headset. The display mode was based on the Fixed 2D mode described previously, but with finer-grained control over the relative position and angle of the display. Users could place the display at a location in front of them, and it would move and rotate with them as they turned their head or moved their body, always maintaining the same relative position.

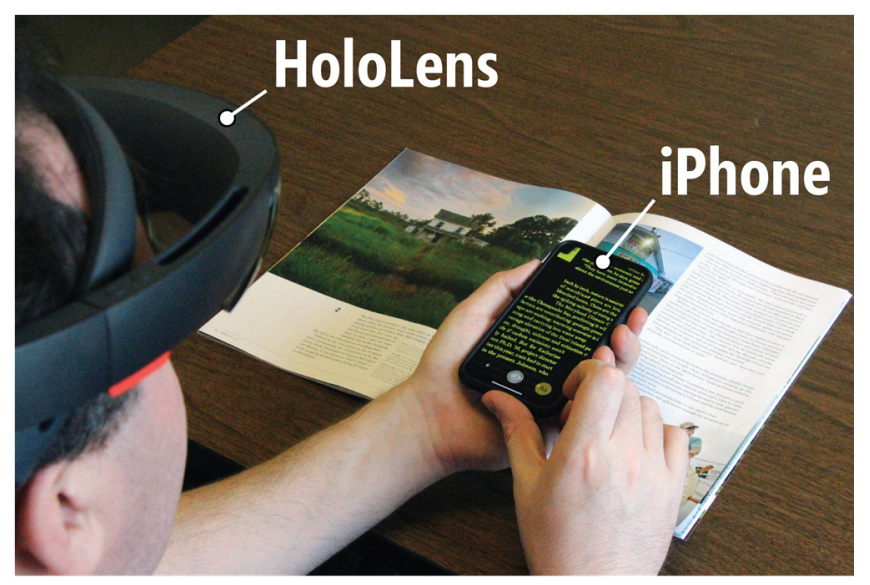

Figure 4. Second prototype AR magnification system using the HoloLens and a hand-held iPhone $X$

- Attached to World. This mode combined the two Fixed 3D designs from the previous study into a single flexible approach that allowed users to position the display in the physical world at any location and angle. As with the earlier designs, the display remained fixed at that physical position as the user moved.

- Attached to Phone. This mode functioned similarly to the Finger Tracked design from the previous prototype. It positioned the display atop the smartphone and the display moved as the user moved the phone, acting like a magnifying glass but with an arbitrarily large virtual display. Users could reposition the display-for example, so that it would be vertical while they held the phone horizontally - but the display would always move to maintain the specified position and rotation relative to the phone.

Aside from the three display designs, we also provided controls for users to adjust the image colors and contrast. As before, we made changes to our designs between sessions. For example, in addition to the black and white color inversion options included for the previous prototype, at the request of P4 we implemented standard white/blue, yellow/blue, grayscale, yellow/black, and red/black filters provided by other digital magnifiers. To address comments from $\mathrm{P} 1$ and $\mathrm{P} 3$, who were the first to try the new prototype, we also added a "freeze frame" mode which allowed users to press a button to stop the video capture and send a fullresolution photograph to the HoloLens for display. Users

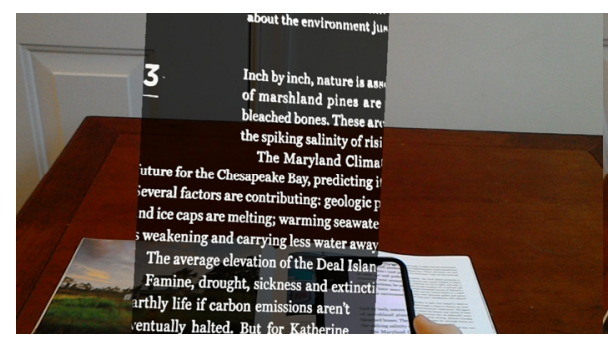

(a) Attached to Headset

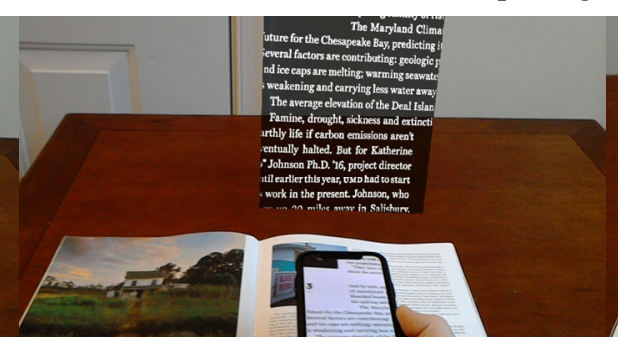

(b) Attached to World

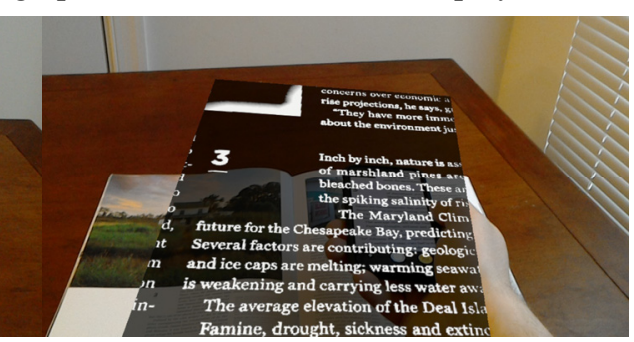

(c) Attached to Phone

Figure 5. Prototype 2 provided three virtual display modes, which were refined versions of the four included with Prototype 1. See the accompanying video figure for a demonstration. 

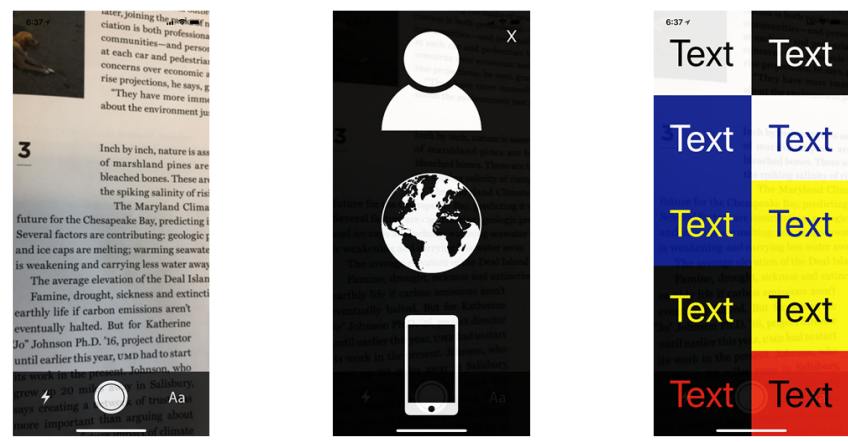

Figure 6. Touchscreen controls on the iPhone prototype. Left to right: main screen, display mode menu, text colors menu.

could then control the image size and position on the AR display as before, but without needing to hold their phone above the target content while reading.

User Input. Touchscreen controls were used for most input (Figure 6), including: double-tapping to open the display mode menu, tapping to select buttons on the screen, pinching to control the size of the virtual display, and sliding to move the display during "freeze frame".

To control the display's 3D position and rotation, we implemented a motion tracking feature using the iPhone's built-in ARKit APIs [40]. The API provides functions to track the phone's $3 \mathrm{D}$ pose relative to its starting location, which we stream to the HoloLens and use to position and rotate the virtual display. Because the iPhone and HoloLens had different internal 3D reference frames, we manually initialized the transformation between the two at the start of each session using a simple procedure that required visually positioning the phone atop a virtual representation. This procedure is overly simplistic, and a more robust method will likely be necessary for long-term use. However, it proved sufficiently reliable for the duration of our study.

Users could move the phone to position the virtual screen as desired for each of the three display modes. In both the Attached to Headset and Attached to World modes, users pressed a finger firmly on the screen until the phone vibrated, moved the virtual screen to the desired location (3D position and rotation), then released their finger. The interaction was slightly different for the Attached to Phone mode, with users first moving the phone to where they wished the screen to be located, then pressing firmly and moving the phone to where they wished to hold it while reading. After lifting their finger off the touchscreen, the virtual screen maintained the offset between the initial and final positions as they continued to move the phone.

\section{Design Sessions}

Six participants tested our updated prototype, comparing the display modes and providing general feedback and open-ended suggestions about their ideal magnification aid.

Participants. We recruited six people with visual impairments (3 male, 3 female, ages 28-68) to participate in design sessions with our updated prototype. P1 and P3 returned from the previous sessions, while four participants had not used our prior prototypes. As with the previous design sessions, the cause and severity of the participants' visual impairments varied (Table 1) but all participants regularly used some type of magnification aid.

Methods. The user sessions were structured similarly to the previous ones. Participants were introduced to the updated prototype and allowed time to explore the options while reading a simple document. After becoming comfortable with the controls, participants then used each of the three display modes in a fully counterbalanced order to read text on a variety of objects, including simple printed documents, magazine articles, mail, and product labels (e.g., box of cereal, pill bottle). After each mode, participants provided feedback on what they liked and disliked. The session closed with a discussion of participants' overall experience using the system, preferred display modes, and feedback on the system and AR magnification in general, and participants' envisioned ideal magnification aid. As with the previous stage, sessions lasted approximately two hours, and participants were compensated $\$ 60$ for time and travel.

Overall Response. Participants were in general more successful and positive about the experience of using this prototype than we had observed with the previous version. The iPhone provided higher quality images and better control over the amount of visible text, and the touchscreen and motion controls provided faster and easier control over enhancements and virtual display settings. Participants were better able to experience the augmented reality aspects of our approach, which most participants found promising. One participant was particularly enthusiastic about the Attached to World design, stating that it was:

"so much better [than her CCTV], you can go down the whole page and read it. Like if I want to read a book or something to my kids, Mommy doesn't have to go line by line. I can read it and keep the flow going. You can read your whole mail in its entirety without it being on your TV." (P4)

She felt that our system provided an experience more like what she remembered before her vision loss with advantages of portability and privacy compared to her existing aids, continuing, "It's everything I need as far as being able to read independently" (P4).

Virtual Display Modes. Participants' display preferences were again mixed, with some participants stating that they liked the flexibility of having multiple designs available and would use different versions depending on the situation. P1 and P3 preferred the Attached to Headset design because they found it easier to focus on the text with fewer variables to consider. In contrast, P4 found that mode to be too distracting, especially when speaking with someone or otherwise multitasking, and preferred the Attached to World design since it functioned, "like a private, portable CCTV that stays where you want it to stay" (P4). P5, P6, 
and P7 saw advantages to all three designs, including the simplicity of the Attached to Headset design, the natural reading experience and ability to multitask with the Attached to World design, and the versatility and intuitive interactions of the Attached to Phone design, especially while away from home (e.g., while shopping). However, all participants found the Attached to Phone design to be more difficult to use than the others for reading longer passages in its current implementation, suggesting that more robust motion tracking and image stabilization are needed to improve the reading experience.

Smartphone Camera. The two participants who had used the previous prototype (P1 and P3) felt that the updated design was an improvement, with a better camera and more usable interactions. However, while the previous design was lightweight and could be used hands-free, the updated design required holding the iPhone steady in midair while reading. All participants found this to be somewhat difficult because of the additional physical effort and shaky image due to unsteady hands. This issue was initially exacerbated by a sometimes slow and uneven frame rate streaming the video from the phone to the HoloLens, which we fixed after the first two sessions, but it also prompted us to add the "freeze frame" feature described above. This feature functioned similarly to existing features on smartphone magnifiers, but with a significantly larger virtual display. Later participants (P4, P6, and P7) liked this feature and found it to be much easier to use than live video when reading longer passages. The issue of image stability could also be addressed in the future by including a portable mount to help hold the phone steady, by adding optical or digital image stabilization, or by integrating the camera and motion controls into a smaller design (e.g., a smartwatch).

The HoloLens Device. While replacing the finger-worn camera with an iPhone camera addressed one aspect of the limited field of view from the previous design (allowing more text to be captured at once), the narrow window that the HoloLens could display was still too small for some participants. This issue was particularly problematic for the two participants with central vision loss, one of whom was completely unable to use the system to read (P5) and one of whom was frustrated by how little of his available vision could be used (P1). In contrast, another participant with tunnel vision found the display to be perfectly sized. The contrast of the HoloLens display also continued to be problematic for some participants, especially for P5 who was unable to see anything on the screen without blocking out all external light. These highly variable results reemphasize the need for customizability.

Summary. Our second prototype system improved on several aspects of the first, with a better camera that could capture a greater amount of text, more polished and robust virtual display options, and efficient controls that allowed users to more easily customize the AR display to fit their needs. Participants were largely positive about our updated design, appreciating the options for customization and noting tradeoffs between the three AR display designs as well as advantages compared to existing technology. The design sessions also helped to identify important features and design elements for future AR magnification aids.

\section{DISCUSSION}

We reflect on the implications of our findings, focusing on ways to support a range of users with different visual impairments and a range of situations.

\section{Overall Experience with 3D Augmented Reality}

Our design sessions explored a novel AR magnification approach. The ability to display content in 3D space enables new interactions that are not possible with handheld devices or head-mounted 2D displays. For example, participants liked that they could use stationary $3 \mathrm{D}$ designs to create and position an arbitrarily large virtual display and then read a full document by turning their head, rather than scanning line by line as with other portable reading aids. Participants also liked how the design allowed them to easily multitask, for example, by turning away from the virtual display to speak with someone, then turning back to continue reading.

However, some participants commented that our approach required more effort to use than other reading aids. These participants preferred the simplicity of designs that fixed the display in 2D in front of their vision (e.g., as in [25]), especially when they are trying to concentrate on the content of what they are reading. Further refinements to our designs and additional time for the participants to practice using the system may have improved their impressions of the system, but it is also possible that more traditional reading aids or simple $2 \mathrm{D}$ image enhancements may work better for some situations or users.

\section{Reflections on Head-mounted AR vs. Handheld Tools}

AR magnification has potential benefits compared to other magnification approaches, but also limitations that must be overcome to offer a compelling alternative to existing aids.

Usability. Smartphone magnifiers are portable and readily available but have limited screen size. Users can hold the phone close to their face to compensate, but that may be uncomfortable for extended periods. Current HMDs do not yet address these issues, but we expect that future iterations will be lighter-weight and provide a perceptually larger display. These physical advances should allow users to read more naturally than with a handheld magnifier.

Flexibility. Our approach separates the camera from the display, allowing users to find a comfortable reading position regardless of the location of the physical world object, and supports customization so that users can adapt the display to their visual needs or context.

Privacy and Discreet Use. Handheld magnifiers and smartphone apps offer portability but may require the user to hold the device close to their face to read, preventing discreet use. While current HMDs attract attention for other 
reasons (unusual, bulky), we expect that future designs will be smaller and less noticeable, and that users will be able to use the magnification aid more privately and discreetly than with a handheld screen - a feature that one participant found particularly appealing.

Ergonomics. Physical strain and fatigue are potential problems for many portable magnification aids [8]. This was also a limitation of our prototypes, but future $A R$ designs could use a smaller wearable camera that can be aimed separately from the display for maximum flexibility and comfort. Participants also noted ergonomic problems with the HoloLens, including weight and eyestrain. These issues are also present to some extent with other head-worn vision enhancement systems. Future HMDs will need to be smaller and more ergonomic with screens designed to support a wide range of vision levels.

\section{Recommended Design and Future Work}

Based on the design sessions, we propose design recommendations and key features for assistive AR devices.

HMD. Participants liked our use of a transparent display that did not block out external vision, unlike most existing HMD systems (e.g., [25,27]). Therefore, an ideal system should use an optical see-through HMD, but in a more lightweight form factor than the HoloLens, with a larger field of view to better support users with limited central vision. However, if contrast cannot be sufficiently improved in future optical see-through HMD devices, a video display that blocks out external light may be a better choice for some low vision users (e.g., P5). Future work should explore alternative display options and evaluate their suitability for different users and contexts.

Camera. Participants valued flexibility, comfort, a wide field of view, and hands-free use, suggesting the need for a wearable camera that can be aimed separately from the display. The finger-worn and handheld smartphone cameras that we tested did not meet these criteria, but neither do the head-worn cameras used in most existing commercial HMD systems (e.g., [27,29]). A head-worn camera should allow for movement and optical zoom independent of the headset so that target content can be captured without requiring users to precisely position their head. Separate motion of the camera and head is also crucial for allowing users to move their head to scan virtual content in $3 \mathrm{D}$, an interaction which participants found intuitive and useful. This design would likely require the ability to detect the content a user is pointing toward so that it can be magnified (e.g., similar to the interaction used by OrCam [14]). Future work should explore these camera options in more depth.

Virtual display. AR magnification systems should include multiple display options to support different users and situations. We encountered tradeoffs between designs, such as the ease of use and attention required, ability to multitask, usefulness for different situations (e.g., reading a long document vs. products in a store), and technical complexity and robustness. The ability to anchor virtual content in 3D space in the physical world can support a more natural and flexible reading experience compared to existing 2D vision enhancement systems, but is also more complicated to implement and may have a steeper learning curve for users. Future work should investigate new AR designs, such as the ability to place multiple displays with different targets or magnification levels, and an option to automatically display enhanced content directly over the text (e.g., on a page or sign).

\section{Limitations}

The HoloLens has a narrow and centrally located field of view (estimated at $30^{\circ} \times 17.5^{\circ}$ ), which did not work well for some users. The translucent "holograms" that the HoloLens displays are also low-contrast, and colors are distorted by the screen material. Two participants could not use the device to read due to these issues, and most of the others mentioned them as limitations as well. We did not evaluate alternative headsets, although we anticipate that future versions of the HoloLens or similar technology will be able to address these issues. Future work should consult vision experts to better assess design requirements and usability for specific visual impairments. While our design sessions were informative and helped identify important design features for AR magnification aids, our study was not controlled, included a relatively small number of participants ( 7 total), and did not quantitatively evaluate usability or reading speed and comprehension. Future work should investigate camera positions and virtual display designs in more depth and directly compare AR magnification aids against existing technology.

\section{CONCLUSION}

This paper explored novel applications of AR to assist low vision users, applying recent technology that can anchor 3D virtual content in the physical world. We explored the AR magnification design space and presented two prototype systems that we evaluated and refined through iterative design sessions with low vision participants. Participants liked the general concept of a head-worn magnification aid for its improved portability, privacy, and ready availability compared to other magnification aids they had used. Participants also identified advantages to our 3D AR approach compared to handheld magnification tools, including a more natural reading experience and the ability to more easily multitask, but also some disadvantages such as a steeper learning curve and limitations of the particular hardware we used. Through our open-ended design and evaluation sessions, we identified several common themes that should inform the design of future AR vision enhancement aids for low vision users.

\section{ACKNOWLEDGMENTS}

We thank our participants, the Maryland State Library for the Blind and Physically Handicapped, Seokbin Kang, Dhruv Jain, and Matt Mauriello. This work was supported by the Office of the Assistant Secretary of Defense for Health Affairs under Award W81XWH-14-1-0617. 


\section{REFERENCES}

1. Ronald T. Azuma. 1997. A Survey of Augmented Reality. Presence: Teleoperators and Virtual Environments 6, 4: 355-385. https://doi.org/ $10.1162 /$ pres.1997.6.4.355

2. American Foundation for the Blind. CCTVs/Video Magnifiers. Retrieved from http://www.afb.org/ prodBrowseCatResults.aspx?CatID $=53$

3. American Foundation for the Blind. Low Vision Optical Devices. Retrieved from www.afb.org/ ProdBrowseCatResults.aspx?CatID $=54$

4. G L Goodrich, E B Mehr, and N C Darling. 1980. Parameters in the use of CCTV's and optical aids. American journal of optometry and physiological optics 57, 12: 881-92. Retrieved March 27, 2018 from http://www.ncbi.nlm.nih.gov/pubmed/6164297

5. Google. Google Glass. Retrieved from https://www.google.com/glass/start/

6. Joan M. Greenbaum and Morten Kyng. 1991. Design at work: cooperative design of computer systems. L. Erlbaum Associates. Retrieved April 16, 2018 from https://dl.acm.org/citation.cfm?id=125470

7. R Harper, L Culham, and C Dickinson. 1999. Head mounted video magnification devices for low vision rehabilitation: a comparison with existing technology. The British journal of ophthalmology 83, 4: 495-500. https://doi.org/10.1136/BJO.83.4.495

8. ChandraM. Harrison. 2004. Low-vision reading aids: reading as a pleasurable experience. Personal and Ubiquitous Computing 8, 3-4: 213-220. https://doi.org/10.1007/s00779-004-0280-0

9. Alex D Hwang and Eli Peli. 2014. An augmentedreality edge enhancement application for Google Glass. Optometry and vision science : official publication of the American Academy of Optometry 91, 8: 1021-30. https://doi.org/10.1097/OPX.0000000000000326

10. Shaun K Kane, Chandrika Jayant, Jacob O Wobbrock, and Richard E Ladner. 2009. Freedom to roam. In Proc. ASSETS 2009, 115. https://doi.org/10.1145/ 1639642.1639663

11. Sreekar Krishna, Greg Little, John Black, and Sethuraman Panchanathan. 2005. A wearable face recognition system for individuals with visual impairments. In Proc. ASSETS 2005, 106. https://doi.org/10.1145/1090785.1090806

12. R W Massof and D L Rickman. 1992. Obstacles encountered in the development of the low vision enhancement system. Optometry and vision science: official publication of the American Academy of Optometry 69, 1: 32-41. Retrieved March 26, 2018 from http://www.ncbi.nlm.nih.gov/pubmed/1371334

13. Suranga Nanayakkara, Roy Shilkrot, Kian Peen Yeo, and Pattie Maes. 2013. EyeRing: A Finger-worn Input
Device for Seamless Interactions with Our

Surroundings. In Proc. AH 2013, 13-20.

https://doi.org/10.1145/2459236.2459240

14. OrCam Technologies Ltd. OrCam - See for Yourself. Retrieved from http://www.orcam.com/

15. Halley Profita, Reem Albaghli, Leah Findlater, Paul Jaeger, and Shaun K. Kane. 2016. The AT Effect: How Disability Affects the Perceived Social Acceptability of Head-Mounted Display Use. In Proc. CHI 2016, 48844895. https://doi.org/10.1145/2858036.2858130

16. Shrinivas Pundlik, Huaqi Yi, Rui Liu, Eli Peli, and Gang Luo. 2016. Magnifying Smartphone Screen using Google Glass for Low-Vision Users. IEEE

Transactions on Neural Systems and Rehabilitation Engineering: 1-1. https://doi.org/10.1109/ TNSRE.2016.2546062

17. Douglas. Schuler and Aki. Namioka. 1993. Participatory design : principles and practices. L. Erlbaum Associates. Retrieved April 16, 2018 from https://dl.acm.org/citation.cfm?id=563076

18. Roy Shilkrot, Jochen Huber, Wong Meng Ee, Pattie Maes, and Suranga Chandima Nanayakkara. 2015. FingerReader. In Proc. CHI 2015, 2363-2372. https://doi.org/10.1145/2702123.2702421

19. Laurindo de Sousa Britto Neto, Vanessa Regina Margareth Lima Maike, Fernando Luiz Koch, Maria Cecília Calani Baranauskas, Anderson de Rezende Rocha, and Siome Klein Goldenstein. 2015. A Wearable Face Recognition System Built into a Smartwatch and the Blind and Low Vision Users. In Proc. ASSETS 2015, 515-528. https://doi.org/10.1007/ 978-3-319-29133-8_25

20. Lee Stearns, Victor DeSouza, Jessica Yin, Leah Findlater, and Jon E. Froehlich. 2017. Augmented Reality Magnification for Low Vision Users with the Microsoft Hololens and a Finger-Worn Camera. In Proc. ASSETS 2017, 361-362. https://doi.org/10.1145/ 3132525.3134812

21. Lee Stearns, Ruofei Du, Uran Oh, Catherine Jou, Leah Findlater, David A. Ross, and Jon E. Froehlich. 2016. Evaluating Haptic and Auditory Directional Guidance to Assist Blind People in Reading Printed Text Using Finger-Mounted Cameras. ACM Transactions on Accessible Computing 9, 1: 1-38. https://doi.org/ $10.1145 / 2914793$

22. Lee Stearns, Uran Oh, Leah Findlater, and Jon E. Froehlich. 2018. TouchCam: Realtime Recognition of Location-Specific On-Body Gestures to Support Users with Visual Impairments. Proceedings of the ACM on Interactive, Mobile, Wearable and Ubiquitous Technologies 1, 4: 1-23. https://doi.org/10.1145/ 3161416

23. Gianni Virgili, Ruthy Acosta, Lori L Grover, Sharon A 
Bentley, and Giovanni Giacomelli. 2013. Reading aids for adults with low vision. Cochrane Database of Systematic Reviews. https://doi.org/10.1002/ 14651858.CD003303.pub3

24. Yuhang Zhao, Michele Hu, Shafeka Hashash, and Shiri Azenkot. 2017. Understanding Low Vision People's Visual Perception on Commercial Augmented Reality Glasses. In Proc. CHI 2017, 4170-4181. https://doi.org/10.1145/3025453.3025949

25. Yuhang Zhao, Sarit Szpiro, and Shiri Azenkot. 2015. ForeSee: A Customizable Head-Mounted Vision Enhancement System for People with Low Vision. In Proc. ASSETS 2015, 239-249. https://doi.org/10.1145/ 2700648.2809865

26. Annuska Zolyomi, Anushree Shukla, and Jaime Snyder. 2017. Technology-Mediated Sight: A Case Study of Early Adopters of a Low Vision Assistive Technology. Proc. ASSETS 2017: 220-229. https://doi.org/10.1145/3132525.3132552

27. eSight Glasses. Retrieved from https://www.esighteyewear.com/technology

28. NuEyes Pro. Retrieved from https://nueyes.com/nueyes-pro/

29. IrisVision. Retrieved from https://irisvision.com/

30. Microsoft Hololens. Retrieved from https://www.microsoft.com/en-us/hololens
31. ClearView + HD. Retrieved from us.optelec.com/ products/hdebbu22ufa-clearview-hd-22-inch-tft-ultraflexible-arm.html

32. DaVinci HD. Retrieved from www.enhancedvision.com/low-vision-productline/davinci-pro.html

33. Ruby Handheld Video Magnifier. Retrieved from http://www.freedomscientific.com/Products/LowVisio $\mathrm{n} /$ Ruby

34. Optelec Compact+ HD. Retrieved from https://us.optelec.com/products/compact-hd.html

35. Apple iOS Accessibility Features. Retrieved from https://www.apple.com/accessibility/iphone/vision/

36. Google Android Accessibility Features. Retrieved from https://support.google.com/accessibility/android/answe r/6006949

37. Microsoft Seeing AI. Retrieved from https://www.microsoft.com/en-us/seeing-ai/

38. Pivothead Seeing AI. Retrieved from http://www.pivothead.com/seeingai/

39. Oculus Rift. Retrieved from https://www.oculus.com/rift/

40. Apple iOS ARKit. Retrieved from https://developer.apple.com/arkit/ 